\title{
The design and modeling of a novel resistive stretch sensor with tunable sensitivity
}

\author{
Amir Firouzeh, Student Member, IEEE, and Jamie Paik, Member, IEEE,
}

\begin{abstract}
Wearable technologies, interactive and safe robotic systems require an effective actuator control that highly depends on the accurate feedback from flexible and dense array of sensors. In this research, we introduce a novel soft stretch sensor design that is applicable to distributed high strain measurements. The proposed sensor design utilizes $2 \mathrm{D}$ fabrication processes to create specific profiles and stretchable mesh patterns in Constantanpolyimide laminate that are scalable and customizable. In addition to the geometrical parameters of the stretchable mesh pattern, the sensitivity in the proposed design is determined by the metal layer profile. Without considerably affecting its mechanical properties and stretchability, the profile design allows an engineering freedom to choose the scope of measurements and the sensitivity. Similar design principle can eventually produce complete sensor / circuit systems where the circuitry and the sensing elements can co-exist in a single metal laminate. In this paper, we describe the sensor design parameters and the sensor model that include the equations of deformation and resistance change as a function of the stretch. We compare the prototype test results to the model prediction. The findings provide guideline to designing customized sensors that satisfies specific size and stretch requirements.
\end{abstract}

Index Terms-stretchable sensor, strain sensor, Constantanpolyimide laminate, low-profile sensor, soft sensor.

\section{INTRODUCTION}

Soft robots in rehabilitation [1] and emergency mitigation [2]-[4] applications aim at making the interaction between the robot and the environment safer and more reliable. However, the inherent softness of these robots complicates the control; feedback from robots' interaction with the environment as well as the body geometry with the minimum interference with its natural compliance becomes necessary for effective control. Although stretchable pressure and temperature sensing arrays for E-skin application have been demonstrated [5], developing stretch sensors that can provide feedback from the geometry of a soft body remains to be a challenging task. This research introduces a sensing method which can easily be integrated into sensing arrays to meet this challenge.

Among possible options for sensing high strain in soft bodies, many rely on measuring strain-induced resistance change either from the piezoresistivity effect in carbon-polymer composites [6], [7] or the geometry change under strain [8]-[10]. These sensors require very little auxiliary equipment which makes them suitable for sensing arrays. Among the resistance based sensors, the robust fabrication of sensors relying on geometry changes in a liquid-metal or ionic liquid-filled microchannels can be challenging which makes the fabrication of

A. Firouzeh \& J. Paik are with the Reconfigurable Robotics Laboratory, Swiss Institute of Technology of Lausanne (EPFL), Lausanne, Switzerland. e-mail: Jamie.paik@epfl.ch . arrays with multiple sensing elements complex. On the other hand, the fabrication process of piezo-resistive sensors is rather easy and scaleable but the main shortcoming of these sensors is their complex response [11], [12]. Moreover, due to the high resistance, these materials are not suitable as conductors since the resistance of the conductive part becomes close to the resistance of the sensing area. This necessitates an extra circuit layer, complicating the integration of these sensors in arrays with many sensing elements.. The other suggested group of sensors are based on measuring capacitance change due to the deformation and shape change in a sandwich of two stretchable electrodes and a stretchable membrane [13]-[15]. Compared to the piezoresistive sensors, these sensors have better transient and drift behavior over time but the choice of membrane and electrode materials for consistent properties in different environmental conditions and with repeated loading cycles is still a challenge.

In this paper, we propose a novel stretch sensing method based on the resistance change of a metal film under strain. A similar concept is also used in conventional metal film strain gauges [16]. Metallic strain sensors are one of the oldest [17] strain sensing methods that are still popular due to their accuracy, robustness, low-cost and customizability. Our research aims to widen the application of this sensing method as stretch sensing element by introducing specific mesh patterns. For strain gauges, the material's elastic strain limits the overall extension of the sensor, thus, the measurement range. The novel mesh pattern and fabrication process allow increasing this extension. This is possible since the mesh structure transforms the large extension in the sensor into bending deformations within the elastic range of the materials. We refer to this extension of the sensor as stretch throughout the manuscript.

Given the limited elastic strain in metals, to make stretchable sensors, we need to design a pattern that transforms a small strain in the metal layer into a large displacement along the sensor. In the literature, there are different methods suggested for transforming the bending motion in the metal film (which causes low strain) into stretch in the sensing element. Meander patterns achieve this by in-plane bending of the metal tracks [18], [19]. Another proposed method uses out-of-the-plane deformation due to a pre-strain in an elastic membrane to create a wavy surface. The amplitude of the waves decreases as the sample is stretched [20], [21]. The third method, previously used by the authors to create stretchable heaters and circuits, uses a mesh pattern consisting of micro beams which twist buckle out-of-plane under strain and bend about their lowest moment of inertia axis [22], [23].

In the meander design the loading condition puts the metal 


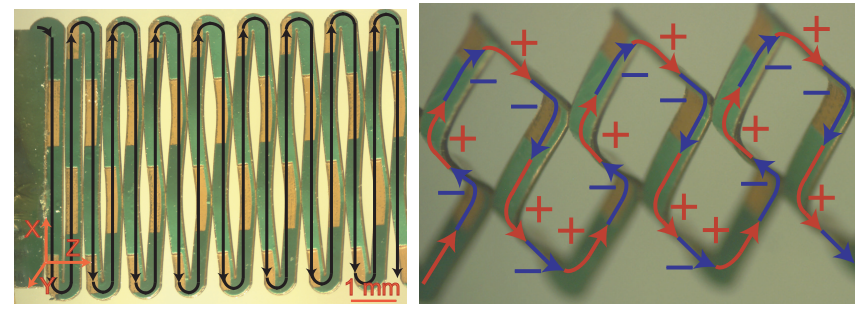

(a)

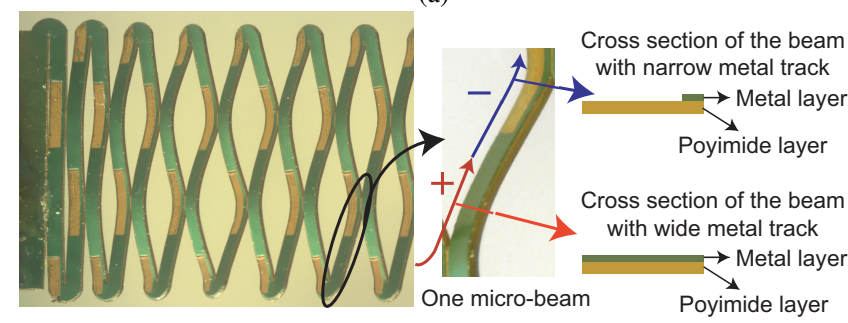

(b)

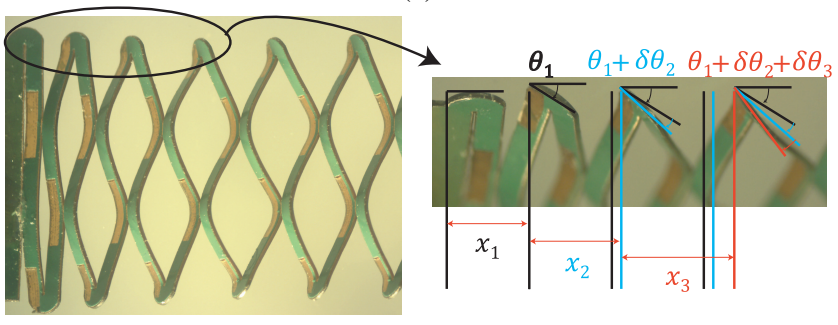

(c)

Fig. 1: Bending deformation of the micro-beams in the sensor pattern as the sample is stretched in three steps (a-c). The electrical current path is shown by the black route in (a). As the sensor is stretched the micro-beams twist out of plane and bend about the axis of lowest moment of inertia which requires less energy leading to positive and negative strain in different sections of the metal layer. One micro beam and different metal profile in sections in tension and compression (b). While the micro-beams in the middle part of the sensor have the same twist angle and similar contribution to the overall stretch in the sensor, the first few columns have slightly different twist angle $\left(\theta_{1}, \theta_{1}+\delta \theta_{2}\right.$, and $\left.\theta_{1}+\delta \theta_{2}+\delta \theta_{3}\right)$ and contribution to the overall stretch $\left(x_{1}, x_{2}\right.$, and $\left.x_{3}\right)$ (c). These figures are from a sample with wider micro-beams $(400 \mu \mathrm{m})$ than the final design $(250 \mu \mathrm{m})$ to better illustrate the sensor design.

layer partly in tension and partly in compression in each cross section. So the overall strain and the resistance change in each cross-section would not be considerable. On the other hand, for the designs that cause out-of-the-plane deformation, the metal layer in each cross section along the conductive path would be either in tension or compression which cause resistance increase and decrease in different cross sections. In this research we make use of this varied loading condition along the conductive path to make stretch sensors, by modulating the metal track width.

Among the two methods that use out-of-plane bending, the fabrication process of the mesh patterns is closer to the conventional printed circuit board (PCB) fabrication process and here we base the sensor design on this pattern. With a design that selectively increases the sensitivity in some parts of a large meshed pattern, the sensing elements and the circuit can be integrated in a thin metal laminate.

The main contributions of this paper are:

- the introduction of a novel stretch sensing method based on the strain in metal films.
- the presentation of the working principle of the sensor and determining the design parameters to selectively control the sensitivity of a stretchable pattern (to have positive, negative or no sensitivity to strain).

- the validation of the sensor reading repeatability in cycles with different loading amplitude and loading rate.

In Section II, the overall design and working principle of the sensors will be presented. In Section III, the design parameters and equations governing stretch and resistance change of the sensing element are presented. In Section IV, the fabrication process of the sensor is elaborated. Section V presents the experimental results and finally, in Section VI, we present conclusions of the work and future steps.

\section{STRETCH OF THE MESH STRUCTURE AND SENSOR'S WORKING PRINCIPLE}

In this manuscript, we present a new stretch sensing method based on the strain induced resistance change in the metal tracks. The sensor is based on a stretchable mesh structure, which was previously used as stretchable heaters and circuit. As will be discussed shortly, the metal layer is in tension and compression in different sections of the stretched mesh structure which makes it rather insensitive to strain (since the effect of the parts in tension and compression cancel each other out). To make the mesh sensitive to stretch, we need to modify the metal track profiles to have different contributions to resistance in sections with positive and negative strain. Effective modulation of the metal track profile requires understanding of the relation between the stretch and the strain distribution in the metal layer. Fig. 1 (a-c) present a sensor as it is stretched. The mesh pattern transforms the linear deformation into bending deformation in the micro-beams. This enables us to reach a large linear deformation without exceeding the maximum elastic strain in each micro-beam. Fig. 1 (c) and (a) also present a side view of the few first columns and couple of middle section columns respectively. Under strain, the microbeams twist out of the plane and bend about the axis with the lowest moment of inertia. As presented in Fig. 1 (c), this twist occurs in the first few columns and from the third column on, the twist angle stays approximately the same and the bending motion is the dominant deformation.

The stretch sensors presented here are made of a thin Constantan film laminated on a polyimide sheet. The bending deformation in the mesh structure puts the metal layer in tension in some parts (marked by + in Fig. 1(a)) of the sensor and in compression in the others (marked by - in Fig. 1(a)). The number of micro-beams and hence the length of the conductive tracks in tension and compression are equal. In the sensor pattern, we modulate the width of the metal tracks in one half (either in the sections with metal layer in tension or in compression) to produce narrower metal tracks with a larger electrical resistance, compared to the electrical resistance of the wide tracks. The electrical resistance of the narrow metal tracks is three times the electrical resistance of the wide metal tracks. In the sensor, the parts with narrow and wide tracks have opposite loading conditions and because of higher initial resistance of the part with narrow tracks, the 
resistance change of this part dominates the overall resistance change of the sensor. Fig. 1 presents an example of a sensor with a narrower track in the half under compression. Under strain, the resistance of this sensor decreases. The radius of curvature and the strain in the metal tracks change along the micro beams. In order to maintain the overall resistance of the sensor as low as possible, the metal tracks in the sections of the sensor without substantial curvature are designed to have the same wide cross section as the parts in tension. So the narrow metal track (in compression) in each beam is shorter than the wide metal track (in tension or without substantial loading). This design reduces the overall resistance of the sensor without much influence on the resistance change and in effect increases the ratio of the resistance change to the initial resistance.

The twist buckling which occurs in the first few columns of the sensor is a phenomenon in which a beam under bending load twists out-of-plane and bends about an axis with a lower moment of inertia. The twist angle is determined so to have the minimum deformation energy. Apart from the first few columns, for the main body of the sensor the dominant deformation is bending along the micro beams. This bending motion for one micro beam is presented in Fig. 1 (b). Fig. 1 (c) presents the twist which occurs in the first few columns. Given the larger number of the beams with dominant bending motion, they have the main influence over the twist angle and resistance change in the sensor. Therefore we model the behavior of the sensor through studying the deformation only in these beams. To determine the relation between the bending deflection of each micro-beam and the stretch, we need to know the twist angle in each micro-beam. Since we only consider bending about the minimum moment of inertia axis, we can estimate the twist angle using geometrical relations. we expect this estimate to be accurate since the moment of inertia about the maximum moment of inertia axis is 2000 times larger. Based on the boundary conditions for each microbeam, we can estimate the twist angle in the middle section of the beam as presented in (1):

$$
\begin{array}{ll}
2 \delta_{z}=2 \delta \sin \theta=D+b(1-\cos \theta) & \\
2 \delta_{y}=2 \delta \cos \theta=b \sin \theta & \frac{\tan \theta \sin \theta}{D+b(1-\cos \theta)}=\frac{1}{b}
\end{array}
$$

In (1), $\delta, \delta z$, and $\delta y$ are the deflection of the beam and its components along $\mathrm{z}$ and $\mathrm{y}$ directions respectively which are

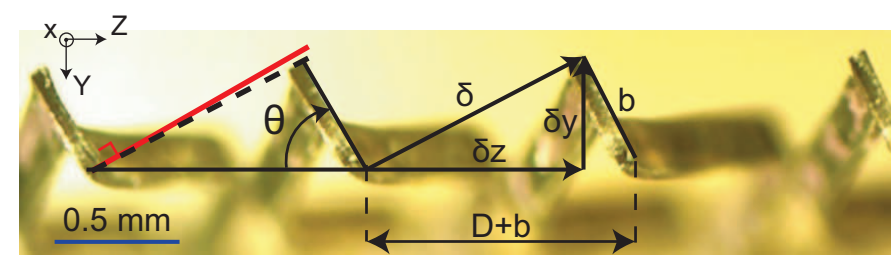

Fig. 2: Twist angle, $\theta$, in the sensor (sensor view along $\mathrm{x}$ axis). To measure this angle as a function of stretch, the sensor was mounted on a linear stage and stretched in $1 \mathrm{~mm}$ steps under a microscope. The small angle between the dashed black and the solid red lines in this figure is caused by the secondary bending about the axis of maximum moment of inertia, $I_{\max }$, which is neglected in this study. depicted in Fig. 2. Here, $b$ is the width of each micro-beam and $\theta$ is the twist angle of the beams. For $D$, the contribution of each micro-beam to the overall stretch, we have:

$$
D=\frac{\Delta x}{N_{\text {columns }}}
$$

where $\Delta x$ is the overall stretch of the sensor and $N_{\text {columns }}$ is the number of columns along the sensor.

Fig. 3 presents the estimated and the measured twist angle as a function of contribution of each micro beam to the elongation $(D)$. To measure the twist angle, we placed the sensor on a micro stage and studied its deformation under a microscope in $1 \mathrm{~mm}$ steps.

We should point out that the deformations considered in (1) are not the only possible deformations to satisfy the boundary condition but these deformations are the most energy efficient and bending about the other axis would require more energy. The estimated value from the model and the measured twist angles match closely. The small discrepancy can be attributed to small bending about the $I_{\max }$ axis. This secondary bending causes the small angle between the dashed black and red lines in Fig. 2. Also as presented in Fig. 1(c), the few first columns have a smaller contribution to the elongation which means that the deflection in the beams in the middle section is slightly underestimated by (2). As will be discussed in Section III, the thickness of the polymer and metal layers were chosen to put the metal layer above the neutral plane; so in each cross section of the micro-beam, the metal layer is entirely in tension or compression depending on the twist direction. The twist direction is not random and is determined by the pattern of the metal layer. For the pattern presented in Fig. 1, the sensor deforms so that the narrow metal tracks would always be in compression and the sensor's resistance decreases as the sensor stretches. For the symmetric sections with a wide metal track, twist can happen on either side and it is the parts with narrow metal tracks that determine the twist direction. Fig. 4 presents a schematic of a micro-beam with a patterned metal layer. It is attached to the left columns on two ends and to the column on the right in the middle section. As the sensor is stretched, the micro-beams can twist about $\mathrm{x}$ axis in both positive and negative directions. If it twists in the positive direction, the moment of inertia of the bending axis $\left(I_{y y}\right)$ increases. As a result this damps the out of plane twist motion. On the other

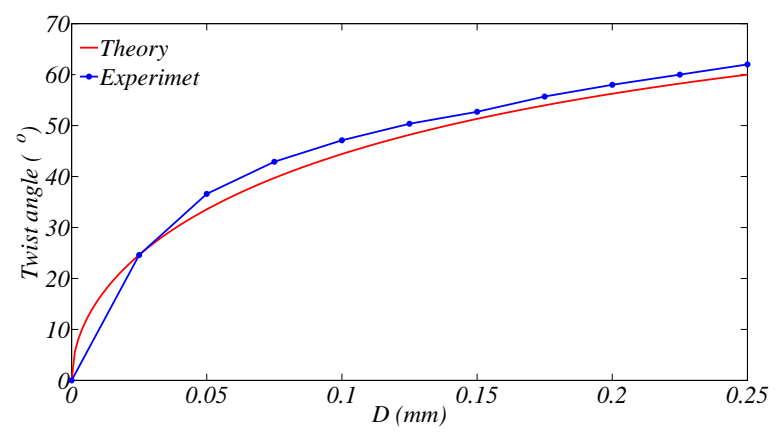

Fig. 3: The twist angle as a function of the contribution of each micro beam to the elongation based on the theory and experiment. 


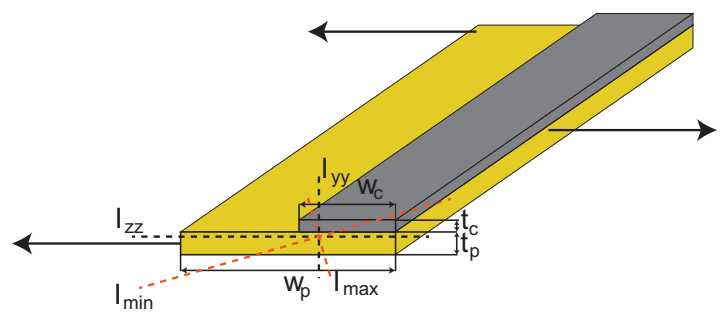

(a)

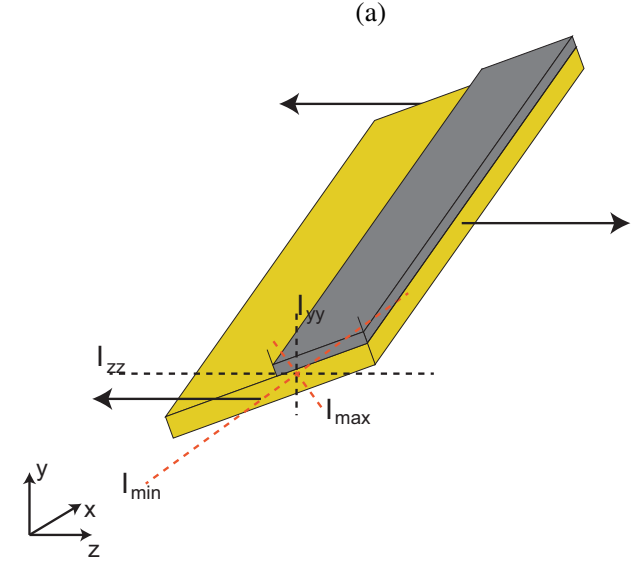

(b)

Fig. 4: The twist in micro-beams occurs to reduce the moment of inertia about the bending axis. The twist direction determines whether the metal layer would be in tension or in compression. While for an unpatterned beam the twist can happen in either directions, in the patterned beam it always happens in a direction that results in a smaller $I_{y y}$. This selective twist happens since the axis of minimum moment of inertia is slightly tilted due to the asymmetric pattern in the metal layer.

hand, if the beam twists in the negative direction of $\mathrm{x}$ axis (Fig. 4 (b)) $I_{y y}$ decreases, which allows bending deformations requiring less energy that would be stable. So the micro-beams with the present pattern always twist in the direction that puts the narrow metal tracks in compression and wide metal tracks in tension. The higher initial resistance in the narrow section causes a larger resistance change compared to the wide section. So the overall resistance change of the sensor is determined by the strain and resistance change in the narrow metal tracks. For the present design with narrow section in compression, the resistance of the sensor decreases under stretch. Accordingly, by etching the metal on the opposite side (mirrored about the y axis), the twist direction would be different and the metal layer with narrow cross section would be on the tension side (hence the overall resistance of the sensor would increase under strain).

\section{SEnSOR DESIGN AND EXPECTED PERFormance}

In this section, we study the design parameters of the sensor in detail and investigate how these parameters affect the mechanical and electrical characteristics of the sensor. The sensor consists of two patterns: a mesh through all the layers and a pattern in the metal layer. The mesh pattern

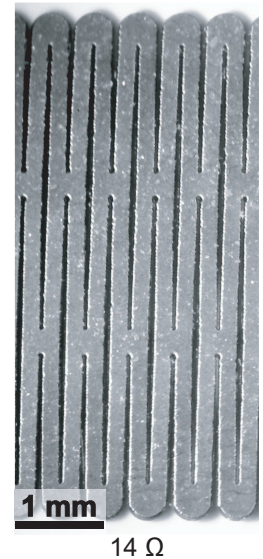

(a)

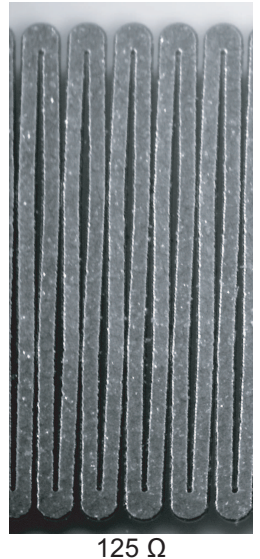

(b)

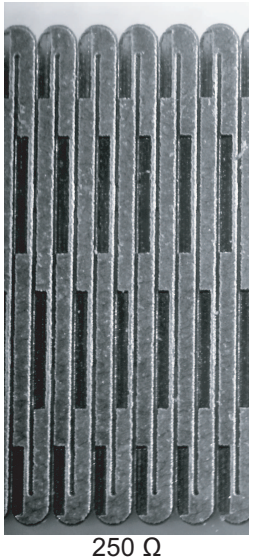

(c)
Fig. 5: The same stretchable structure can have different electrical resistance and sensitivity to stretch. The mesh structure with very low resistance can be used as a circuit to make the electrical connections between different parts (a). The serpentine path which has a large electrical resistance (10 times the mesh structure) but low sensitivity to stretch (given its symmetric electrical path) can be used as a voltage divider for reading the sensors and canceling out the thermal effects (b). Finally the complete pattern which selectively increases the contribution of that part of the sensor in compression exhibits high sensitivity to strain(c). Thickness of the Constantan film used to check the electrical properties of different design was $10 \mu \mathrm{m}$. The resistance is reported for 80 columns of micro-beams.

(see Fig. 5(a)) makes the sensors stretchable. The pattern in the metal layer consists of two parts, a serpentine path (see Fig. 5(b)) which is designed on top of the mesh pattern, and an asymmetric pattern (see Fig. 5(c)) which selectively makes narrower metal tracks in the sections under tension or compression. The role of the serpentine path is to increase the resistance and hence the overall resistance change of the sensor.

A sample with just the mesh pattern in Fig. 5(a) can be used as a circuit with low resistance and sensitivity to stretch which can provide connection between different sensing elements. Etching the metal along the serpentine pattern on the same mesh pattern produces a resistor with a high resistance but a low sensitivity as presented in Fig. 5(b) which can be used as a voltage divider resistance for reading the sensor and canceling out the thermal effects. As mentioned, to make the pattern of Fig. 5(b) sensitive to strain, the metal tracks in tension or compression are selectively exposed and etched to become narrower (hence having a higher contribution to the final resistance change). This leaves us with the asymmetric pattern of Fig. 5(c) with narrower metal tracks in compression which we will use as a stretch sensor.

To have the maximum range of stretch, it is imperative to choose the minimum thickness for the sensor to reduce the strain as the micro-beams bend. The sensor is made of a laminate of Constantan on polyimide. For the samples used to refine the design and those used to study the electrical properties of different patterns we used $10 \mu \mathrm{m}$ Constantan film. For the final prototype we used $5 \mu \mathrm{m}$ Constantan film. As will be discussed using thinner Constantan film also increases the sensitivity. In this Section, we base our design on the thinner film. We chose the thickness of the polyimide layer 
to place the neutral plane somewhere in the polyimide to have the whole metal layer in each cross-section in tension or compression. Fig. 4 presents a schematic of the beam's cross section and the relative position of the metal layer and neutral plane. Considering simple bending, to place the neutral plane at the intersection of the Constantan and polyimide, the ratio of the thickness of the two layers should satisfy the following relation:

$$
(E S)_{\text {Constantan }}=(E S)_{\text {polyimide }} \rightarrow k^{2}=\frac{E_{\text {Constantan }}}{E_{\text {Polyimide }}}
$$

In (3), $S$ is the first moment of area, $E$ is the modulus of elasticity, and $k$ is the ratio of the thickness of the polyimide to Constantan. Based on (3), the thickness of the poyimide layer for $5 \mu \mathrm{m}$ Constantan film should be $40 \mu \mathrm{m}$. Based on the availability, we used Pyralux LF0110 (from Dupont) composed of $25 \mu \mathrm{m}$ polyimide and $25 \mu \mathrm{m}$ acrylic adhesive layer as the substrate for laminating Constantan. In the rest of this paper, we approximate the properties of the adhesive layer by polyimide properties and model the laminate as a film composed of $5 \mu \mathrm{m}$ Constantan and $50 \mu \mathrm{m}$ polyimide. In this laminate, the neutral plane will be in the polyimide layer and $1.2 \mu \mathrm{m}$ below the intersection with the metal layer.

If the metal layer undergoes a large strain, in repeated cycles, the sensor readings would show a drift. Also a plastic deformation in any of the layers will cause a bias in the sensor reading. Accordingly, the maximum range of stretch in the sensor is dependent on the strain in the laminate as the micro-beams bend. Neglecting the first few columns where the twist motion happens, we study the strain in each microbeam considering simple bending under concentrated load at one end. Fig. 6 presents the loading condition on each microbeam. The maximum deflection in each beam is limited by the minimum allowed radius of curvature $\left(\rho_{\min }\right)$ which is governed by the maximum elastic strain $\left(\varepsilon_{\max }\right)$ as presented in (4).

$$
\rho_{\min }=\frac{y}{\varepsilon_{\max }}
$$

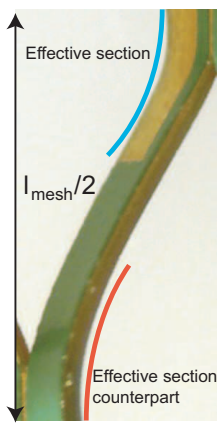

(a)

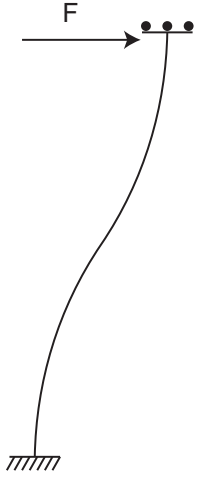

(b) (c)

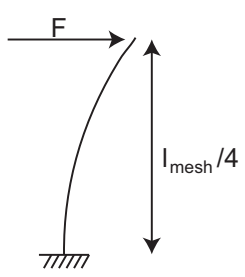

Fig. 6: Bending deformation in one of the micro-beams that makes up the mesh structure (as presented in Fig. 1(c)). The section with narrow metal track and effective resistance change and its counter part with wide metal track are highlighted in (a). The schematic of the equivalent loading condition (b). Based on the symmetry in the beam, we can study the bending deformation in half of the actual beam (c).
In (4), $y$ is the distance from the neutral plane. The maximum elastic strain is dependent on the properties of each layer. Considering the loading condition introduced in Fig. 6, we have the following relation governing the beam's deflection:

$$
\delta_{\max }=\frac{\left(l_{\text {mesh }} / 4\right)^{2}}{3 \rho_{\min }}
$$

For a desired range of stretch, one can choose the design parameters $\left(l_{\text {mesh }}, b\right.$, and the number of columns) so as to keep the radius of curvature above $\rho_{\min }$. We should point out that changing the beam width would also affect the twist angle of the micro-beams and its contribution to the overall stretch according to (1). The mechanical properties of the laminate used in the sensors is dependent on the properties of each individual layer and is also influenced by the fabrication processes such as heat press lamination and laser micro machining which makes its estimation from properties of individual layers complicated. Here, we will determine the allowed range of motion using experimental data in Section V. Through the experimental data and (5) one can estimate the maximum allowed strain in the laminate which can be used as a guideline for designing sensors with specific requirements in future.

In the rest of this Section, we study the sensitivity of the sensor to stretch. The resistance change in the stretched sensor originates from the strain in the metal layer. Considering the gauge factor of 2 for Constantan, the resistance change in the effective part and its counter part in Fig. 6(a), $\delta R$, is estimated using the mean strain in the beam as follows:

$$
\delta R=2 R \overline{\bar{\varepsilon}}
$$

where $R$ is the electrical resistance of the corresponding portions of the micro beam and $\overline{\bar{\varepsilon}}$ is the mean strain in the metal layer. To evaluate the mean strain, we used the following equation for strain, $\varepsilon$, in each cross section:

$$
\varepsilon=\frac{y}{C} \varepsilon_{m}
$$

where $\mathrm{C}$ is the distance of the outer layer of the metal to the neutral plane. $\varepsilon_{m}$ is the minimum or the maximum strain in the metal layer (for a beam in compression or in tension respectively). Integrating the above equation gives us the following relation for the average strain in each cross section $\left(\bar{\varepsilon}_{\text {section }}\right)$ :

$$
\bar{\varepsilon}_{\text {section }}=\frac{1}{t_{c}} \frac{\left(t_{c}+y_{c}\right)^{2}-y_{c}^{2}}{2\left(t_{c}+y_{c}\right)} \varepsilon_{m}
$$

where $y_{C}$ and $t_{C}$ are the distance of the Constantan-polyimide intersection from the neutral plane and the thickness of the Constantan layer respectively. $y_{c}$ in (6) is different for the sections with narrow and wide metal tracks and is equal to $5.3 \mu \mathrm{m}$ and $1.2 \mu \mathrm{m}$, respectively. It is important to note that in this study we neglected the initial tilt of the minimum inertia axis in sections with narrow metal track (see Fig. 4). Considering the ratio of the width of the micro-beam and the thickness of the Constantan layer this tilt angle is small and neglecting it would not affect the result significantly. 
As described, we estimated the deformation of each microbeam as a beam in simple bending and under concentrated load at one end. For this loading condition, the curvature, $1 / \rho$, changes linearly along the beam between zero and the maximum curvature, $1 / \rho_{\min }$, (given the linear distribution of the moment along the beam). So on the average, the strain along the whole length would be the mean of the strain of the beginning and end of each part. Combining (5), (6), and (8) will give the following expression for the resistance change of the effective section and its counterpart in each micro beam. These are highlighted in Fig. 6(a).

$$
\begin{aligned}
& \delta R=2 R \overline{\bar{\varepsilon}} \\
& =2 R \frac{1}{2 t_{c}} \frac{\left(t_{c}+y_{c}\right)^{2}-y_{c}^{2}}{2\left(t_{c}+y_{c}\right)} \frac{3 C}{\left(l_{\text {mesh }} / 4\right)^{2}} \delta\left(1+\frac{\delta l}{l_{\text {mesh }} / 4}\right)
\end{aligned}
$$

The term $\delta l / l_{\text {mesh }} / 4$ accounts for the difference between the length of the part in tension and compression. The value for the present design is $0.15 . y_{c}$ and $R$ in this equation are different for effective section with narrow metal track and its counterpart with wide cross-section. These differences are the reason for the overall resistance change.

We should point out that due to the different moment of inertia in the sections of the micro-beams with narrow and wide metal tracks, the displacement of the mesh pattern will not be entirely symmetric and sections of the beams with narrow and wide metal tracks have slightly different deflections. The difference, however, is negligible as observed in Fig. 6(b). (9) suggests a linear relation between resistance and deflection of the micro-beams. The only non-linearity arises from the relation between the sensor's stretch and the deflection in the micro-beams as suggested in (1). This is specifically important in small deflections where the rate of the twist angle change is large.

The overall resistance change in the sensor, $\Delta R$, is the sum of the resistance change in the effective sections in compression, $\delta R_{C}$, and their counterpart in tension, $\delta R_{T}$ :

$$
\Delta R=\delta R_{T}+\delta R_{C}
$$

To evaluate (9) and (10), we require the values of the design parameters that depend on the application. For the experimental studies in this research, we used a design with parameters determined based on the experience and limitations dictated by the fabrication process. The slit size in the mesh pattern, $l_{\text {mesh }}$, in the case study is $3.45 \mathrm{~mm}$. In this pattern, the beam width is $250 \mu \mathrm{m}$, the track size in the wide section is $230 \mu \mathrm{m}$ and in the narrow section is $90 \mu \mathrm{m}$. As it will be discussed in Section IV, the metal track width is smaller than the designed value because of the removal of the metal from under the protective layer. So the resistance of the sensors are larger than the resistance expected from the design parameters of the tracks. In order to evaluate $R_{T}$ and $R_{C}$, we fabricated one sample with the asymmetric pattern and the other with only the serpentine pattern and measured their resistances. We found the resistance of the effective part in compression and its counterpart in tension to be $210 \Omega$ and $660 \Omega$, respectively.

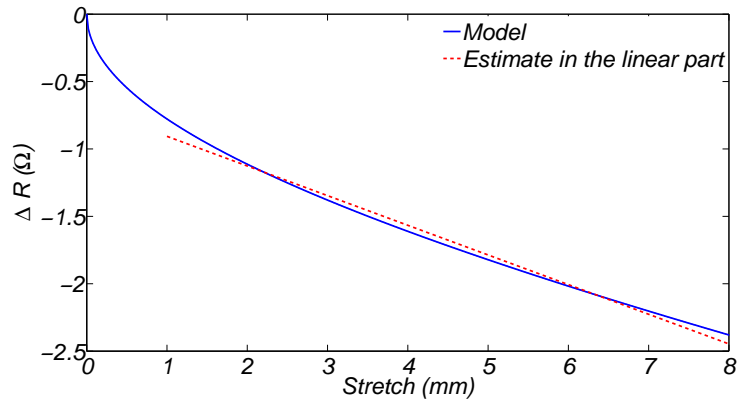

Fig. 7: Estimated resistance change as a function of the stretch in the sensor. The response is quite linear (the slope is $-0.22 \Omega / \mathrm{mm}$ ) aside from the first $1 \mathrm{~mm}$ of stretch, which corresponds to the region with considerable and nonlinear change in the twist angle (see Fig. 3). We have calculated the resistance change for a sample with $20 \mathrm{~mm}$ sensing length equivalent to 80 columns of micro-beams so the result would be comparable with the experimental result of Section V.

Using these values along with (9) and (10), we can estimate the resistance change in the sensor.

Fig. 7 presents the estimated resistance change as a function of the stretch of the sensor. The nonlinearity in the response is caused by the nonlinear relation between stretch in sensor and deflection in the micro-beams as presented in (1). The response of the sensor over $1 \mathrm{~mm}$ stretch can be closely estimated by a linear function with $R^{2}$ of 0.992 . As will be discussed in Section V, due to the residual deformation in the mesh structure in the experiments we will be in the linear part of this plot. The slope of the line fitted to this part is $-0.22 \Omega / \mathrm{mm}$.

\section{FABRICATION PROCESS OF THE SENSOR}

The stretch sensors introduced here are thin and highly customizable. To fabricate these sensors we engrave, machine and chemically process the surface of the laminate using 2D fabrication methods that have been developed for micro system manufacturing. The fabrication of the sensors starts with laminating Constantan (CU040200 from Goodfellow) and Pyralux films (LF0110 from Dupont) $5 \mu \mathrm{m}$ and $50 \mu \mathrm{m}$ thick, respectively, under heat $\left(190{ }^{\circ} \mathrm{C}\right)$ and pressure (approximately $2.5 \mathrm{Mpa}$ ). Constantan was chosen because of its negligible

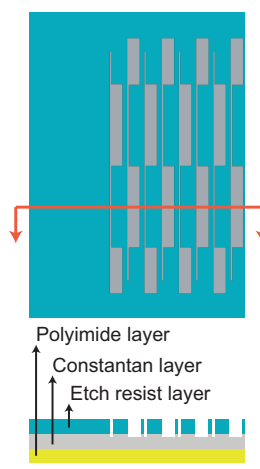

(a)

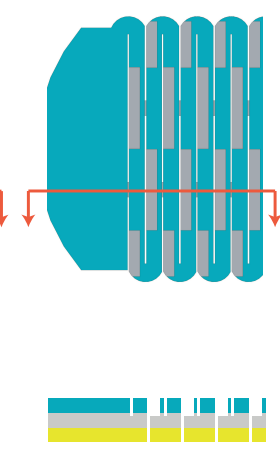

(b)
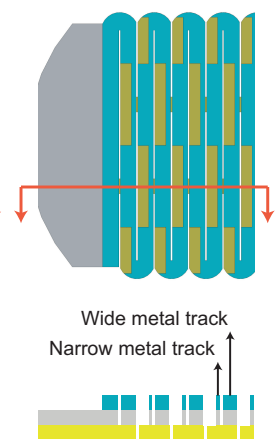

(c)
Fig. 8: Three steps of the stretch sensor fabrication: exposing the metal layer in the desired areas (a), micro-machining the mesh pattern and the outline of the sensor (b), and etching the metal in the exposed areas to create the desired pattern in the metal layer (c). 
bulk resistance sensitivity to temperature. After lamination, the surface of the Constantan layer is covered with etch resist. After curing the resist layer, the sensor pattern is cut through this layer to expose the metal layer (see Fig. 8(a)). To engrave the desired area, we use a laser micro machining system to machine lines $20 \mu \mathrm{m}$ apart. Engraving the surface in the first step of fabrication (see Fig. 8(a)) is not completely uniform mainly because of the uneven surface of the sample. This result in a non-uniform wet etching process. Therefore, to ensure that all tracks remain connected, we keep the minimum track width in the engraving process. In our experiments, the minimum reproducible track size was determined to be $90 \mu \mathrm{m}$; used in the sensor design here.

After engraving the sensor pattern in the resist layer, the mesh pattern is cut through all layers (see Fig. 8(b)) and the outline of the sensor is cut and it is removed from the plate. In the next step, the metal layer in the exposed parts is cleared in ferric chloride etching solution (see Fig. 8(c)). This step of the process is very sensitive and over-etching damages the tracks. On the other hand, if the metal in the exposed parts is not removed completely, it causes irregularity in sensor reading under strain. After this step, the resist on the electrode parts are cleared and wires are connected to the electrodes using conductive epoxy (CW2460 from Chemtronics). Removing the etch resist layer in the final product damages the adhesive layer bonding the Constantan and polyimide layers. This resist layer does not change the mechanical properties by much, given its thickness while it provides electrical insulation. So in the end we leave the resist layer on the metal surface.

We can design the pattern for sensors to have either increasing or decreasing resistance under strain by making the metal path in parts in tension or compression narrower, respectively. In the study of the twist angle and bending deformation in the micro-beams in the previous section we considered symmetric deformation. For the sensor with the narrow sections in compression as presented in Fig. 1 the deformation in beams with the narrow and wide metal paths do not differ much. For the sensors with increasing resistance though, the beams with narrow tracks bend more with the result that the metal layer yields and breaks in smaller elongations. This is caused by the different behavior of the Constantan and bonding layer in tension and compression. So In this work we focused on the more robust pattern with decreasing resistance that places the narrow tracks in compression.

\section{RESULTS AND DISCUSSION}

In this Section, we investigate the response of the stretch sensor prototype under loading. We study the quasi-static response of the sensor in detail and examine the effect of loading rate on the sensor response.

\section{A. Quasi static response of the sensor}

To study the quasi-static response we first compare the sensitivity of a sample with the complete pattern including the asymmetric pattern in the metal layer, similar to Fig. 5(c), and a sample with the same mesh and serpentine pattern but without the asymmetric pattern in the metal layer, similar to Fig. 5(b). The goal is to confirm the proposed working principle of the sensor and to study the possibility of using the sample with the symmetric pattern as voltage divider resistance. The sensors fabricated for studying the pattern are made out of laminate with $10 \mu \mathrm{m}$ thick Constantan. The resistance of the symmetric sample is $125 \Omega$. Etching the metal layer in the desired areas increases the resistance to $250 \Omega$. The difference between the two is the effective resistance of the sensor. Fig. 9 presents the result of the stretch tests on these two samples when stretched in $500 \mu \mathrm{m}$ increments up to $2.5 \mathrm{~mm}$ and back. The meshed length of the samples in both cases is $20 \mathrm{~mm}$. As expected, the resistance in the sample with the complete pattern decreases under load. The results show high coherence in sensor reading over 50 cycles. Although the overall resistance change is not high compared to the initial resistance for the sensor, the coherent sensor reading in 50 cycles with mean standard deviation of $1.7 \%$ of the overall resistance change confirms acceptable accuracy. For the sample with the symmetric pattern, we expected no resistance change under strain but among the three samples tested, the resistance always increased with stretch in the sensor. This rules out to some extent the random uneven wet etching of the beams in tension and compression as the reason for the increase in resistance under strain. This increase in resistance could be because of the uneven gauge factor of the Constantan in tension and compression which is also reported for the commercial film strain gauges. The resistance change for the symmetric sample is less than $10 \%$ of the resistance change of the asymmetric sample. We have tested the same samples up to $3.5 \mathrm{~mm}$ stretch but since the readings started to drift in repeated cycles (indicating overloading of the metal layer), we presented the result of the experiment with $2.5 \mathrm{~mm}$ stretch which corresponds to $12.5 \%$ stretch in the length of the sensing area.

One way to increase the sensitivity of the sensors without surpassing the maximum allowed strain or limiting the maximum allowed stretch in the sensor is using thinner Constantan

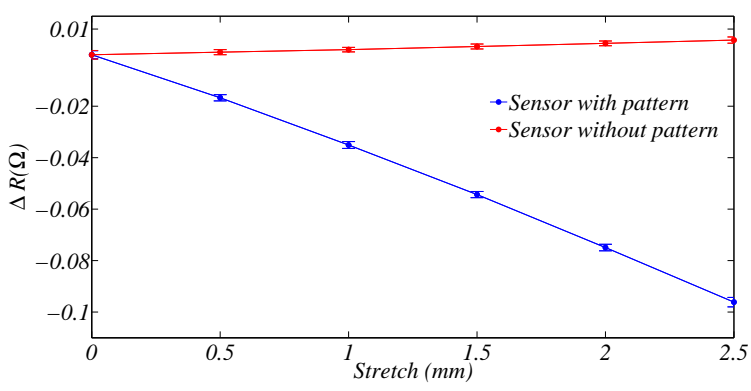

Fig. 9: The result of 50 stretch cycles for a sample with the complete pattern and a sample with just the serpentine and mesh patterns. The active length of both samples is $20 \mathrm{~mm}$. As expected, the resistance of the sample with complete pattern decreases under strain and shows high sensitivity to strain and a good repeatability with mean standard deviation of $1.7 \%$ (of the whole range of resistance change) in 50 loading cycles. For the control sample without the asymmetric pattern, we expected no resistance change, but the tests on symmetric samples repeatedly showed a small increase in the resistance under strain. The resistances of the sample with full pattern and the control sample where 250 and $125 \Omega$ respectively. 
layer in the laminate. This increases the average magnitude of the strain in the metal layer (9) by placing the neutral plane further away from the metal layer and inside the polyimide layer. Also by making the laminate thinner, the maximum strain in the laminate for a given stretch decreases. We fabricated a sensor with $5 \mu \mathrm{m}$ Constantan and the same polymer layer used in the thicker samples ( $25 \mu \mathrm{m}$ polyimide and $25 \mu \mathrm{m}$ adhesive). Fig. 10 presents the result of the quasi static tests on this sensor. This result confirms that by using a thinner Constantan layer, a higher sensitivity and a larger repeatable range of stretch can be achieved. We tested the sensor with different maximum stretch amplitudes with $500 \mu \mathrm{m}$ increments. Here, the goal was to establish the maximum allowed stretch for the sample and to ensure that the sensor reading is repeatable in different loading amplitudes (as discussed in [12], for many soft sensors, the amplitude related drift is a big drawback). We tested the sensor in 20 cycles for each amplitude. The mean cycle for each test is presented in Fig. 10 which shows coherence. At $4 \mathrm{~mm}$ stretch, the sensor reading at zero deviates from its initial value, which corresponds to the plastic deformation in the sensor. By increasing the pre-strain in the sensor, we could use it for larger stretches but for the present design parameters, $4 \mathrm{~mm}$ (the start of the plastic deformation) is advisable as the allowed range of stretch (equivalent to $20 \%$ of the sensor length). To design sensors to meet other requirements, one can use this maximum deformation along equations (1) - (5).

Contrary to the initial expectation from the model (see Fig. 7), the response of the sensor is almost linear in its entire range of deformation. This discrepancy is due to the unrecoverable twist in the sensor. After stretching the sensor for the first cycle, part of the twist angle and deformation in the sensor will not be recovered (to model this, one needs to study the twist buckling of the beam which is beyond the scope of this paper). This residual strain shifts the response of the sensor to the linear part of Fig. 7. We should point out that the sensor was conditioned with few cycles of medium amplitude prior to the experiment.

The slope of the resistance change for small stretch in Fig. 10 is less than the rest of the measurement points. For

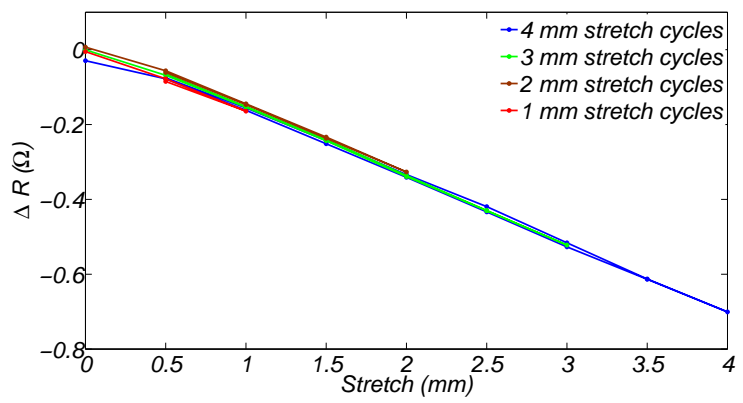

Fig. 10: Resistance change as a function of stretch in the sensor. The plot presents the average of 20 cycles for four amplitude of loading $1-4 \mathrm{~mm}$. For the present design, stretch up to $4 \mathrm{~mm}, 20 \%$ of the length, causes no damage and plastic deformation in the sensor. The response of the sensor can be estimated by a line with the slope of $-0.18 \Omega / \mathrm{mm}$ ). The maximum hysteresis loop width is $2 \%(0.014 \Omega)$ of the maximum resistance change and is of the same order of magnitude as the standard deviation. the maximum deformation curve $(4 \mathrm{~mm})$ this slope change becomes even more significant. We can attribute this smaller slope to plastic deformation in the sensor. Ignoring the first point, the slope of the resistance change in this figure is $-0.18 \Omega / \mathrm{mm}$. This is comparable but smaller than the estimated value from theory $(-0.22 \Omega / m m)$. One reason for this could be the different gauge factor for the metal in tension and compression which was mentioned as the reason for the resistance increase in the symmetric sample in Fig. 9.

Gauge factor of the sensor defined in (11) is the parameter reported in different studies which corresponds to the sensitivity of the sensor.

$$
G F=\frac{\Delta R / R}{\Delta L / L}
$$

The gauge factor of the presented sensor is lower compared to the other methods presented in the literature ( 0.004 for the presented sensor compared with 3.6 for liquid metal sensors [8] and 0.06-0.8 for carbon nanotube sensor [7]). Lower gauge factor does not necessarily translate into lower accuracy for the sensor and RMSE for the presented sensor (bellow 2\%) is comparable to the error for the other sensing methods (RMSE of $6.4 \%$ for [8] and 3\% creep error [7]). However, the lower gauge factor means that we need to take careful measures in reducing sources of noise and other sources of error (such as temperature variation).

Due to the inherent visco-elastic behavior of the polymers, sensors with polymeric components often exhibit a different response as the loading rate changes which is caused by their transient response. This can cause problems in applications with fast and varying loading rates. We present here the response of the sensor in two different loading rates with maximum amplitude to study the significance of this effect. For the fast loading, we used a maximum speed of the linear stage resulting in $65 \mathrm{~s} / \mathrm{cycle}$ and for the slow rate we used a deformation rate 4 times slower.

\section{B. Loading rate effect}

Fig. 11 shows the result of this test. Though not comprehensive (since faster loading rates were not possible with the current test setup), this result is promising. The average difference between the readings from the two loading conditions

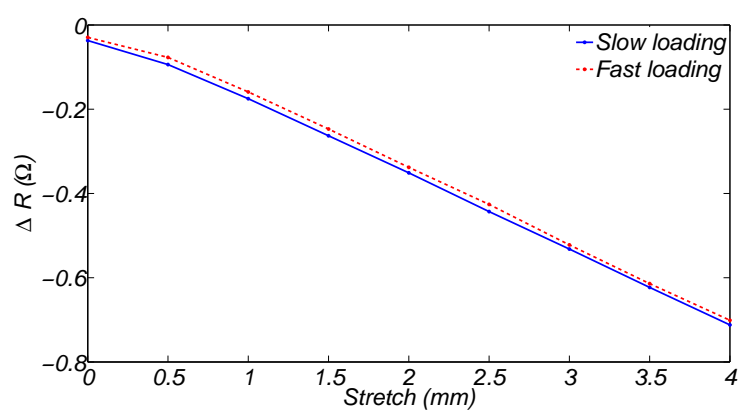

Fig. 11: The average of 20 cycles of stretch for the same sample with two different loading rates (65 and $205 \mathrm{~s} /$ cycle). The graph shows small difference between the average cycles $(1.5 \%)$ which is in the range of the standard deviation of the sensor reading. 
is $1.5 \%$ which is around the same value for the standard deviation of the sensors.

\section{CONCLUSION AND FUTURE WORK}

We introduced a novel sensing concept based on the strain in metal film for measuring stretch. Being thin and complaint, this sensing method can be used in different emerging soft and wearable robots. The possibility to control the sensitivity by modulating the profile of the metal layer on a same mesh structure, enables us to produce stretchable surfaces with integrated sensing elements and circuitry out of a low profile Constantan-polyimide laminate.

The sensitivity and accessible range of motion of the stretchable sensors are determined by the strain in the metal layer. To this effect, we studied the relation between stretch and strain in the metal layer. The theoretical relationships presented, combined with the experimental results on the case study provide us with a guideline to determine design parameters for a desired maximum stretch to produce the maximum sensitivity while having the strain in the materials limited to the maximum elastic strain.

The experimental results of the stretch sensors demonstrated the possibility of controlling the stretch sensitivity of samples having the same mesh structure. The response of the sensor was confirmed to be linear in its whole range of motion. The resistance change per millimeter of stretch in the sensor's length for the design presented here was $-0.18 \Omega / m m$ which is comparable with the value predicted by the model, $-0.22 \Omega / \mathrm{mm}$. One reason for the difference could be the asymmetric behavior of the material in tension and in compression which causes an increase in the resistance of a symmetric pattern under load. The maximum stretch for this pattern was $20 \%$ which may be extended by changing the design parameters. The sensor readings were coherent and repeatable with a mean standard deviation below $2 \%$ for the tested sensors. We also confirmed that the response of the sensor is the same regardless of the maximum range of motion while staying within the elastic range. To study the dynamic response of the sensor, two different loading rates were tested which confirmed negligible dynamic effects. The maximum loading rate was limited by the test setup and studying dynamic effects in a faster loading conditions will require more tests. The temperature dependency of the resistance is another aspect of the sensor response that requires further studies. Optimizing the mechanical design of the sensor for expanding the elastic stretch range is also of interest. This would require more in depth study of the twist buckle motion of the beams and the stress concentration in the metal tracks due to the profile patterns. We believe with further refinements, the novel sensing method proposed in this research can be used in a variety of wearable robotics, human robot interaction and soft robotics where compliance and low thickness are of specific importance

\section{ACKNOWLEDGMENT}

This research is supported by the Swiss National Centers for Competence in Research (NCCR) in Robotics.

\section{REFERENCES}

[1] S. Yun Seong, S. Yi, R. van den Brand, J. von Zitzewitz, S. Micera, G. Courtine, and J. Paik, "Soft robot for gait rehabilitation of spinalized rodents," in Intelligent Robots and Systems (IROS), 2013 IEEE/RSJ International Conference on, pp. 971-976.

[2] R. F. Shepherd, F. Ilievski, W. Choi, S. A. Morin, A. A. Stokes, A. D. Mazzeo, X. Chen, M. Wang, and G. M. Whitesides, "Multigait soft robot," Proceedings of the National Academy of Sciences, vol. 108, no. 51, pp. 20400-20403, 2011.

[3] A. Firouzeh and J. Paik, "Robogami: A fully integrated low-profile robotic origami," Journal of Mechanisms and Robotics, vol. 7, no. 2, pp. 021 009-021 009, 2015, 10.1115/1.4029491.

[4] A. Firouzeh, M. Ozmaeian, A. Alasty, and A. Irajizad, "An ipmc-made deformable-ring-like robot," Smart Materials and Structures, vol. 21, no. 6, p. 065011, 2012.

[5] T. Someya, Y. Kato, T. Sekitani, S. Iba, Y. Noguchi, Y. Murase, H. Kawaguchi, and T. Sakurai, "Conformable, flexible, large-area networks of pressure and thermal sensors with organic transistor active matrixes," Proceedings of the National Academy of Sciences of the United States of America, vol. 102, no. 35, pp. 12 321-12 325, 2005.

[6] L. Wang and J. Li, "A piezoresistive flounder element based on conductive polymer composite," Sensors and Actuators A: Physical, vol. 216, no. 0, pp. 214-222, 2014.

[7] T. Yamada, Y. Hayamizu, Y. Yamamoto, Y. Yomogida, A. IzadiNajafabadi, D. N. Futaba, and K. Hata, "A stretchable carbon nanotube strain sensor for human-motion detection," Nat Nano, vol. 6, no. 5, pp. 296-301, 2011, 10.1038/nnano.2011.36.

[8] Y.-L. Park, B.-R. Chen, and R. J. Wood, "Design and fabrication of soft artificial skin using embedded microchannels and liquid conductors," Sensors Journal, IEEE, vol. 12, no. 8, pp. 2711-2718, 2012.

[9] R. K. Kramer, C. Majidi, R. Sahai, and R. J. Wood, "Soft curvature sensors for joint angle proprioception," in Intelligent Robots and Systems (IROS), 2011 IEEE/RSJ International Conference on, pp. 1919-1926.

[10] J. B. Chossat, P. Yong-Lae, R. J. Wood, and V. Duchaine, "A soft strain sensor based on ionic and metal liquids," Sensors Journal, IEEE, vol. 13, no. 9, pp. 3405-3414, 2013.

[11] M. A. Lacasse, V. Duchaine, and C. Gosselin, "Characterization of the electrical resistance of carbon-black-filled silicone: Application to a flexible and stretchable robot skin," in Robotics and Automation (ICRA), 2010 IEEE International Conference on, pp. 4842-4848.

[12] A. Firouzeh, A. F. Amon-Junior, and J. Paik, "Soft piezoresistive sensor model and characterization with varying design parameters," Sensors and Actuators A: Physical, vol. 233, pp. 158 - 168, 2015.

[13] D. P. J. Cotton, I. M. Graz, and S. P. Lacour, "A multifunctional capacitive sensor for stretchable electronic skins," Sensors Journal, IEEE, vol. 9, no. 12, pp. 2008-2009, 2009.

[14] S. Rosset, B. M. O’Brien, T. Gisby, D. Xu, H. R. Shea, and I. A. Anderson, "Tunable grating with active feedback," vol. 8687, pp. $86872 \mathrm{~F}-$ 86872F-11, 10.1117/12.2009358.

[15] B. O'Brien, T. Gisby, and I. A. Anderson, "Stretch sensors for human body motion," vol. 9056, pp. 905 618-905 618-9, 10.1117/12.2046143.

[16] E. Jonathan, C. Jack, and L. Chang, "Development of polyimide flexible tactile sensor skin," Journal of Micromechanics and Microengineering, vol. 13, no. 3, p. 359, 2003.

[17] L. Ball, "Strain gauge," p. US2556132 A, Jun 5, 1951.

[18] S. Petsch, R. Rix, B. Khatri, S. Schuhladen, P. Mller, R. Zentel, and H. Zappe, "Smart artificial muscle actuators: Liquid crystal elastomers with integrated temperature feedback," Sensors and Actuators A: Physical, no. 0.

[19] J. Vanfleteren, M. Gonzalez, F. Bossuyt, Y.-Y. Hsu, T. Vervust, I. De Wolf, and M. Jablonski, "Printed circuit board technology inspired stretchable circuits," MRS Bulletin, vol. 37, no. 03, pp. 254-260, 2012.

[20] J. Song, H. Jiang, Y. Huang, and J. A. Rogers, "Mechanics of stretchable inorganic electronic materials," Journal of Vacuum Science and Technology; A, vol. 27, no. 5, pp. 1107-1125, 2009.

[21] S. P. Lacour, J. Jones, S. Wagner, T. Li, and Z. Suo, "Stretchable interconnects for elastic electronic surfaces," Proceedings of the IEEE, vol. 93, no. 8, pp. 1459-1467, 2005.

[22] A. Firouzeh, S. S. Mirrazavi Salehian, A. Billard, and J. Paik, "An under actuated robotic arm with adjustable stiffness shape memory polymer joints," in Robotics and Automation (ICRA), 2015 IEEE International Conference on, May 2015, pp. 2536-2543.

[23] J. K. Paik, R. K. Kramer, and R. J. Wood, "Stretchable circuits and sensors for robotic origami," in Intelligent Robots and Systems (IROS), 2011 IEEE/RSJ International Conference on, pp. 414-420. 